International Journal of Current Advanced Research

ISSN: O: 2319-6475, ISSN: P: 2319 - 6505, Impact Factor: SJIF: 5.995

Available Online at www.journalijcar.org

Volume 6; Issue 4; April 2017; Page No. 3364-3367

DOI: http://dx.doi.org/10.24327/ijcar.2017.3367.0274

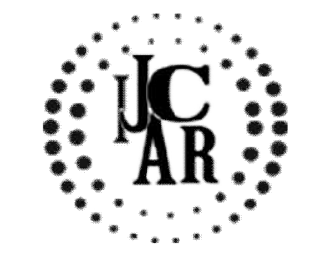

Research Article

\title{
KNOWLEDGE AND AWARNESS ON DIABETIC KETOACIDOSIS AMONG DENTAL STUDENTS
}

\author{
Palak Mayur Shah., Ashish R. Jain and Dhanraj
}

Saveetha Dental College and Hospitals Chennai - 44

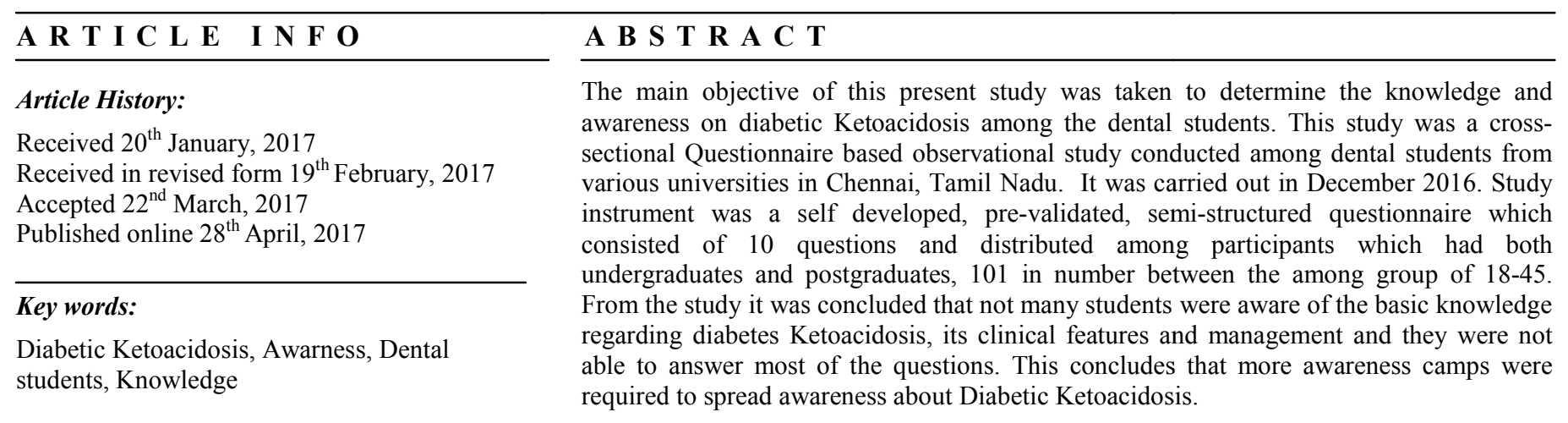

Copyright $\bigcirc 2017$ Palak Mayur Shah., Ashish R. Jain and Dhanraj. This is an open access article distributed under the Creative Commons

Attribution License, which permits unrestricted use, distribution, and reproduction in any medium, provided the original work is properly cited.

\section{INTRODUCTION}

Diabetic ketoacidosis (DKA) is a life-threatening condition that is caused when cells in the body are unable to receive glucose, as there is not enough production of insulin. When the sugar cannot get into the cells, it stays in the blood. The kidneys filters some of the sugar from the blood and remove it from the body through urine.As to because the cells cannot receive sugar for energy, the body begins to break down fat and muscle for energy. When this happens, ketones, or fatty acids, are produced and they enter into the blood circulation, causing the chemical imbalance (metabolic acidosis) called diabetic ketoacidosis.[1] Diabetes ketoacidosis is associated with 10 to $30 \%$ decrease in life expectancy mainly due to its complications associated and die at an earlier age than non-diabetics. These complications are divided into acute and chronic complications. Chronic complications are further divided into micro-vascular and macro-vascular complications. Diabetic ketoacidosis is a preventable condition that usually has a satisfactory outcome. However, the potential for a poor outcome and even death demands early and aggressive treatment. Insulin administration, rehydration, and electrolyte replacement are the mainstays of treatment. [2,3]

The incidence is difficult to establish, ranging from 4.6 to 8 episodes per 1000 patients with diabetes in population-based studies from the USA [1]. In England, more than $11 \%$ of people with Type 1 diabetes had an episode of diabetic ketoacidosis in the years between 2004 and 2009 [2].

*Corresponding author: Palak Mayur Shah

Saveetha Dental College and Hospitals Chennai - 44
It remains a life-threatening condition despite improvements in diabetes care [4]. Mortality in the UK from diabetic ketoacidosis is low at approximately $2 \%$ [5], but each death is potentially avoidable. There is a need to improve patient education and attendance at clinics and to raise awareness of diabetic ketoacidosis and its management amongst healthcare professionals. The mortality rate remains high in developing countries and among non-hospitalized patients. This illustrates the importance of early diagnosis and implementation of effective prevention programmes.

\section{MATERIALS AND METHODS}

A sample size of 101 was calculated, the responders were from the age group of 18-45, where assuming a prevalence of $70 \%$ for knowledge and awareness of Diabetic Ketoacidosis (DKA) among dental students. Convenience sampling was used to draw the sample for this survey. This cross-sectional study was conducted on dental students from various universities in Chennai, Tamil Nadu in Southern India during December 2016. The participants were assured that the outcome would not be used for performance appraisal of the students. To maintain the confidentiality, the personal information of the participants was not disclosed (only gender of the students was mentioned). The questionnaire consisted of 10 questions, including the gender based questions. The purpose of the study was explained to the respondents and informed verbal consent was taken from all the respondents. The questions consist of general information on Diabetic Ketoacidosis, regarding signs, symptoms and management. It also contained question on screening and occurrence of DKA in family. The respondents were given sufficient time to fill 
the questionnaire. Every participant returned the questionnaire and there were no non responders. The responses of the participants were evaluated by giving scores for multiple options questions and correct and incorrect responses for others.

\section{RESULTS}

The following results have been displayed according to the questionnaires which were given to the responders. Their respective answers were evaluated and analysed in the form of various statistical analysis.

Have you ever heard about Diabetic ketoacidosis (DKA)?

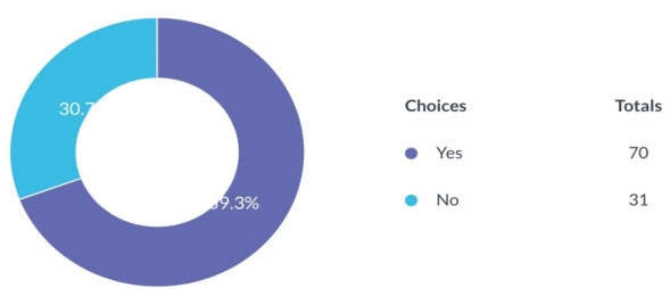

$$
\text { If yes, where have you heard about it? }
$$
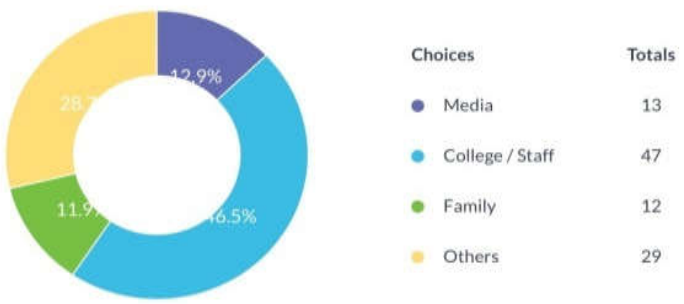

According to the questionnaire, we can see that about $70 \%$ of the responders have heard about Diabetic ketoacidosis and very few are unaware of the terminology and its effects. 47 of the responders have heard about this disease from college/staff, 12 of them from other family members. Media have helped 13 responders, and 29 of them have derived this information from other sources.

Q5 Does anybody in your family has it?

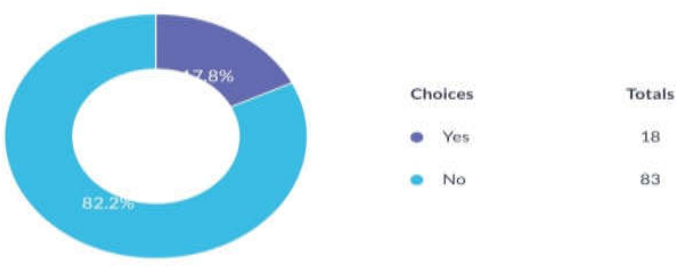

Q6 If yes, how many have it?

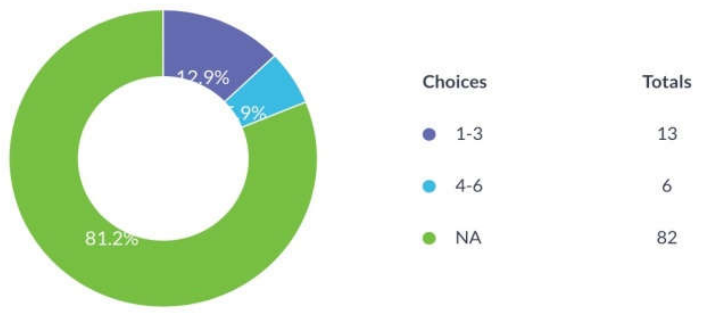

When questioned whether any family member suffered from the disease, $83 \%$ of the responders had a negative response. The positive answers were further asked to mention the number of family members suffering from the disease. $12.9 \%$ choose approximately $1-3$ family members. About $5.9 \%$ have 4-6 members suffering from the disease.

$$
\text { Q7 Signs and symptoms of DKA? }
$$
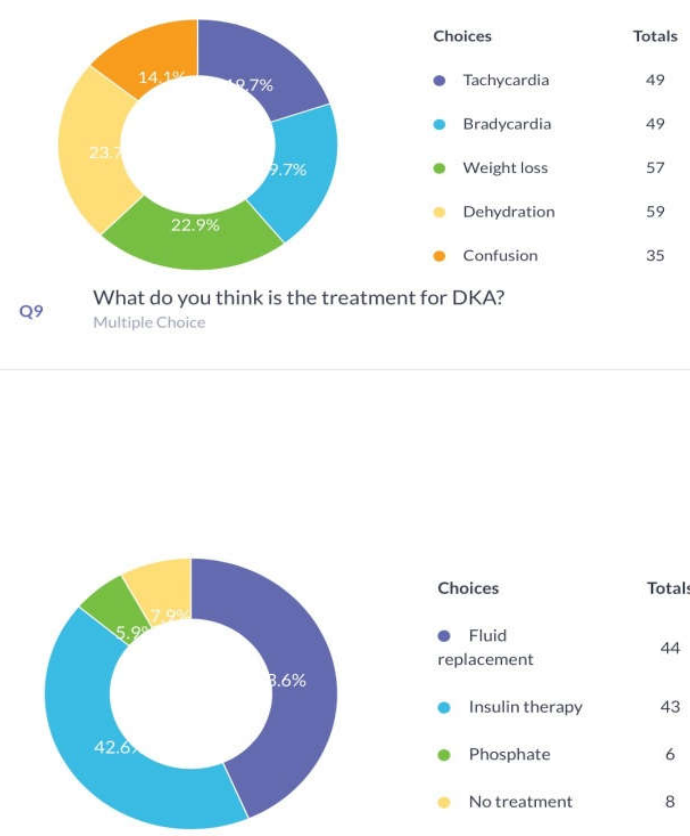

Diabetic ketoacidosis have various signs and symptoms associated with it. The responders were told to choose the appropriate symptoms they believe occur during the disease.

An equal of $39.4 \%$ believe that Tachycardia and Bradycardia are the main symptoms caused. Various other symptoms such as weight loss, dehydration and confusion are believed to be associated by $60.7 \%$ responders. Major treatment of Diabetic ketoacidosis is known to a wide population such as Fluid replacement and Insulin therapy are known to 87 responders. Phosphate treatment which is not used widely is known only to 6 of them and 8 think there is no cure to this disease. 
Q10 What do you think are the complications occurred due to the treatme DKA?
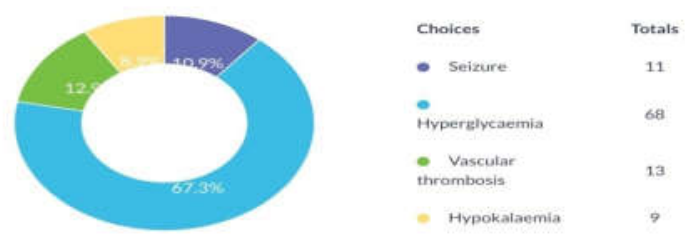

Diabetic ketoacidosis have complications which occur due to the treatment. Hyperglycemia, Vascular thrombosis, seizure and hypokalaemia are some common complications. $67.3 \%$, $12.9 \%, 10.9 \%, 8.9 \%$ responders respectively choose the given complications.

\section{Questionnaire}

1. Gender:

a) Male b) Female

2. What is your age group?

a) $18-24$ b) $25-34$ c) $35-44$ d) $45-54$

3. Have you heard about Diabetic Ketoacidosis (DKA)?

a) Yes b) No

4. If yes, where have you heard about it?

a) Media b) College/Staff c) Family d) Others

5. Does anybody in your family have it?

a) Yes b) No

6. If yes, how many have it?

a) 1-3 b) 4-6 c) N/A

7. Signs and symptoms of DKA?

a) Tachycardia b) Bradycardia c) Weight Loss d) Dehydration e) Confusion

8. Do you think there is a relationship between DKA and dentistry?

a) Yes b) No

9. What do you think are the treatment for DKA?

a) Fluid replacement b) Insulin therapy c) Phosphate e) No treatment

10. What do you think are the complications occurred due to the treatment of DKA?

a) Seizure b) Hyperglycemia c) Vascular thrombosis d) Hypokalemia

\section{DISCUSSION}

In our best knowledge this is the first study done for evaluating the knowledge regarding diabetic ketoacidosis among dental students and its importance. The participants in the study were BDS students who have completed the theory aspect of physiology, pathology and pharmacology regarding diabetes and are going to be exposed to the clinical settings where they will find a number of diabetic patients presenting with both acute and chronic complications. From the analysis of the data regarding general awareness of diabetes, there was a statistically significant difference in between two groups in questions related to classical symptoms.

Regarding awareness of world diabetes day only $15.07 \%$ of the total respondents were able to give correct answer. So even though DM is known to everyone as it is the important topic in every medical, general, social area, there was a lack in awareness among the dental students. This study assesses the awareness and knowledge of DKA among the various universities in Chennai. Students who had heard of osteoporosis were younger and better educated. General knowledge on DKA was low in our study group, with the average score of 5.52 being $22.6 \%$ of the possible maximum score. [6] So even though DM is known to everyone as it is the important topic in every medical, general, social area, there was a lack in awareness among the dental students. [1]

However, all undergraduate students cannot be expected to witness and participate in the management of all critical illness. Many students do not see DKA in real-life patients and, while they may have the knowledge from lecture based educational methods, they may not have the necessary diagnostic and management skills as expected of a practising doctor. The scenario is mapped to core educational domains (Figure 3) allowing students to practise key tasks involved in the diagnosis and management of DKA. The structured debrief is a key component to the simulation experience allowing for appraisal of knowledge, skills and attitudes shown during the exercise. It allows for further tutor facilitated discussion regarding potential pitfalls in management of DKA including potassium prescribing and replacement, fluid management, levels of care and management of cerebral oedema. 9 One particular educational need identified from our experience is that of insulin prescribing and selecting the correct formulation of insulin for use in DKA.(8)

Diabetic ketoacidosis is a serious complication of diabetes and the leading cause of death of people with diabetes. Delay in the diagnosis and treatment of DKA increases morbidity and can lead to mortality, usually from cerebral edema. The initial diagnosis of DKA may not be straightforward. Some patients present with flu-like symptoms, and the diagnosis can be missed, especially during flu season. Prevention of severe metabolic decompensation through sick-day management remains a main reason of comprehensive diabetes treatment. Even in developed countries, patients have died from DKA because of undiagnosed diabetes in recent years. Increased awareness of diabetes symptoms should be reinforced among general practitioners, pediatricians, and in schools. Simple interventions like posters and educational leaflets circulated to physician offices and schools may help reduce DKA at diagnosis through earlier recognition of symptoms. [7]

\section{CONCLUSION}

From the study it was concluded that not many students were aware of the basic knowledge regarding diabetes Ketoacidosis, its clinical features and management and they were not able to answer most of the questions. This concludes that more awareness camps were required to spread awareness about Diabetic Ketoacidosis.

\section{References}

Self care practice and its predictors among adults with Diabetes Mellitus on follow up at Nekemte Hospital, West Ethopia. Tadele Amente 1 (MSc), World journal of Medicine and Science. Vol. 2, No. 3, March 2014

Knowledge of Diabetic complications in patients with Diabetes Mellitus, J Ayub Med Coll Abbottabad 2015; 27(2): $563-568$ 
Management of diabetic ketoacidosis in childhood. Diabetes Ther. 2010 Dec; 1(2): 103-120.

Joint British Diabetes Societies guideline for the management of diabetic ketoacidosis. Diabetic Medicine. Savage MW, et.al. 2011 May 1; 28(5):50815.

Diabetic ketoacidosis (DKA) in Birmingham, UK, 20002009: an evaluation of risk factors for recurrence and mortality. Wright J, et al. Br J Diabetes Vasc Dis 2009; 9: 278-282.

\section{How to cite this article:}

Palak Mayur Shah et al (2017) ' Knowledge And Awarness On Diabetic Ketoacidosis Among Dental Students', International Journal of Current Advanced Research, 06(04), pp. 3364-3367.

DOI: http://dx.doi.org/10.24327/ijcar.2017.3367.0274 